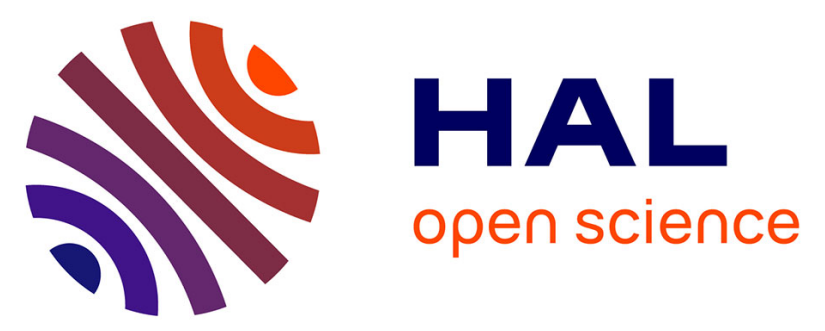

\title{
Comportement de frittés et de films d'oxyde de titane en présence d'atmosphères gazeuses, hors équilibre thermodynamique, en régime stationnaire ; application aux capteurs résistifs d'oxygène
}

R. Jerisian, Joël Gautron, J. Loup

\section{- To cite this version:}

R. Jerisian, Joël Gautron, J. Loup. Comportement de frittés et de films d'oxyde de titane en présence d'atmosphères gazeuses, hors équilibre thermodynamique, en régime stationnaire ; application aux capteurs résistifs d'oxygène. Journal de Physique III, 1992, 2 (4), pp.679-699. 10.1051/jp3:1992153 . jpa-00248775

\section{HAL Id: jpa-00248775 \\ https://hal.science/jpa-00248775}

Submitted on 1 Jan 1992

HAL is a multi-disciplinary open access archive for the deposit and dissemination of scientific research documents, whether they are published or not. The documents may come from teaching and research institutions in France or abroad, or from public or private research centers.
L'archive ouverte pluridisciplinaire HAL, est destinée au dépôt et à la diffusion de documents scientifiques de niveau recherche, publiés ou non, émanant des établissements d'enseignement et de recherche français ou étrangers, des laboratoires publics ou privés. 
Classification

Physics Abstracts

$86.30 \mathrm{G}-81-07.20 \mathrm{~K}$

\title{
Comportement de frittés et de films d'oxyde de titane en présence d'atmosphères gazeuses, hors équilibre thermodynamique, en régime stationnaire ; application aux capteurs résistifs d'oxygène
}

\author{
R. Jerisian, J. Gautron et J. P. Loup \\ Laboratoire de Physique Electronique et Thermodynamique des Oxydes, UFR Sciences et \\ Techniques, Parc de Grandmont, 37200 Tours, France
}

(Reçu le 30 novembre 1990, révisé le 25 octobre 1991, accepté le 27 décembre 1991)

\begin{abstract}
Résumé. - La non-stæchiométrie de l'oxyde de titane est étudiée, dans le domaine de température 870-1 100 K, en présence d'atmosphères hors équilibre thermodynamique, composées de $\mathrm{CO}, \mathrm{CO}_{2}, \mathrm{O}_{2}$, Ar. La résistance électrique de différentes structures massives, poreuses ou en couches est mesurée, en régime stationnaire, en fonction du débit des gaz ; ces atmosphères sont d'autant plus éloignées de l'équilibre que le débit est élevé. On montre que les variations de résistance des échantillons, en fonction du débit, dépendent du domaine de pression partielle d'oxygène, de la température et de la structure des échantillons. La sensibilité à l'oxygène est accrue par une morphologie qui, à l'échelle macroscopique, favorise la diffusion de l'oxygène et qui, à l'échelle microscopique, permet la création de barrière de potentiel aux joints de grains. En milieu oxydant, à $870 \mathrm{~K}$, un mécanisme de volume qui met en jeu la cinétique des échanges avec la phase gazeuse, détermine une variation de résistance en $P_{\mathrm{O}_{2}}^{1 / 4} / P_{\mathrm{CO}}^{1 / 2}$. En milieu réducteur, à la même température, la non-stoechiométrie en volume de l'oxyde est fixée par le couple redox $\mathrm{CO} / \mathrm{CO}_{2}$ comme si l'atmosphère était en équilibre thermodynamique. Mais l'abondance des électrons de conduction, favorise la chimisorption de l'oxygène qui contrôle la résistance des couches selon une loi en $P_{\mathrm{O}_{2}}^{1 / 2}$ en créant des barrières de potentiel à la surface des grains. Des modèles, permettant le calcul du potentiel de surface ainsi que du taux de recouvrement, sont développés; la comparaison des modèles indique une diffusion relativement lente des lacunes d'oxygène à $870 \mathrm{~K}$. A $1100 \mathrm{~K}$, l'atmosphère tend à être à l'équilibre thermodynamique : i) en atmosphère oxydante, tous les capteurs sont en équilibre avec la pression partielle d'oxygène; ii) en atmosphère réductrice, dominée par le couple redox $\mathrm{CO} / \mathrm{CO}_{2}$, seules les couches sont sensibles à la présence de traces d'oxygène.
\end{abstract}

Abstract. - Oxygen resistive titanium dioxide sensors are useful to control combustion but their studies raise fundamental problems. The non-stoichiometry of titanium dioxide is studied in the $870-1100 \mathrm{~K}$ temperature range by using, out of equilibrium, gas mixture flow of $\mathrm{CO}$, $\mathrm{CO}_{2}, \mathrm{O}_{2}$ and Ar. The electrical resistance of sintered samples (dense or porous) or films is measured in steady-state conditions as a function of the gaz flow rate. The deviation to equilibrium increases with the flow rate. The sample resistance is a function of the flow rate, oxygen partial pressure, temperature and microstructure of the specimen. The sensitivity to oxygen is increased by fast oxygen diffusion through the bulk creating a potential barrier at the surface of the grains. Under oxidizing conditions at $870 \mathrm{~K}$, a bulk mechanism determines the 
resistance variation which is proportional to $P_{\mathrm{O}_{2}}^{1 / 4} / P_{\mathrm{CO}}^{1 / 2}$. If reducing conditions are applied, in the same conditions of temperature, the oxide develops a bulk non-stoichiometry which is controlled by the redox couple $\mathrm{CO} / \mathrm{CO}_{2}$ assuming thermodynamical equilibrium. However the large number of conducting electrons favours an oxygen chemisorption, creating potential barriers at the surface of the grains. Accordingly the film resistance is a $P_{\mathrm{O}_{2}}^{1 / 2}$ function. The surface potential and coverage rate are calculated through several theoretical models; their comparison allows to conclude in a relatively slow diffusion of oxygen vacancies at $870 \mathrm{~K}$. At $1100 \mathrm{~K}$, the gaz mixture is rather close to thermodynamical equilibrium : in oxidizing medium the sample is equilibrated with oxygen, under $\mathrm{CO} / \mathrm{CO}_{2}$ reducing conditions film sensors are only sensitive to oxygen traces.

\section{Introduction.}

La non-stæchiométrie des oxydes est étudiée depuis de nombreuses années lorsqu'ils sont en équilibre thermodynamique avec l'atmosphère qui les entoure. Le besoin de développer des capteurs de gaz conduit à mettre de tels oxydes dans des conditions de non-équilibre; ainsi se trouvent posés d'intéressants problèmes fondamentaux. Nous présenterons des mesures de résistance électrique de différentes structures d'oxyde de titane, de 870 à $1100 \mathrm{~K}$, en fonction $\mathrm{du}$ taux de déséquilibre d'atmosphères composées de $\mathrm{CO}, \mathrm{CO}_{2}, \mathrm{O}_{2}, \mathrm{Ar}$, le taux de déséquilibre décroissant lorsque la température augmente et croissant avec le débit. De multiples études ont tenté d'expliquer le fonctionnement des capteurs de gaz pour la combustion air/fuel ou pour des applications particulières (détection du $\mathrm{CO}$, etc...). Ces capteurs sont, soit de type galvanique [1-7], soit de type résistif [8-13]. Nous nous limiterons à des atmosphères composées de $\mathrm{CO}, \mathrm{CO}_{2}, \mathrm{O}_{2}$, Ar et à une étude succincte du $1^{\text {er }}$ cas, car seul le $2^{\mathrm{e}}$ fait intervenir la non-stœchiométrie des oxydes, objet de notre étude.

Pour des capteurs galvaniques (zircones stabilisées), Fouletier puis Salaun [6, 7] utilisent, afin d'interpréter la variation du potentiel de la sonde en fonction du débit de l'atmosphère hors d'équilibre, la notion de potentiel mixte; ce potentiel se situe entre les tensions d'équilibre des deux systèmes $\mathrm{O}_{2} / \mathrm{O}_{\mathrm{O}}^{x}$ et $\mathrm{CO}_{2} / \mathrm{CO}$, et dépend de la cinétique de chacune des réactions :

$$
\begin{gathered}
\mathrm{O}_{\mathrm{O}}^{x} \rightleftarrows \frac{1}{2} \mathrm{O}_{2}(\mathrm{~g})+\mathrm{V}_{\mathrm{O}}^{2}+2 \mathrm{e}^{\prime} \\
\mathrm{CO}(\mathrm{g})+\mathrm{O}_{\mathrm{O}}^{x} \rightleftarrows \mathrm{CO}_{2}(\mathrm{~g})+\mathrm{V}_{\mathrm{O}}^{2}+2 \mathrm{e}^{\prime}
\end{gathered}
$$

Le symbole $\mathrm{O}_{\mathrm{O}}^{x}$ représente un oxygène neutre dans sa position normale du réseau et $\mathrm{V}_{\mathrm{O}}^{2 *}$ une lacune d'oxygène deux fois ionisée. Dans ce modèle, les espèces adsorbées à la surface du solide ne sont pas en équilibre thermodynamique entre elles.

Quant aux capteurs résistifs :

- Logothetis [8] considère que le volume et la surface du $\mathrm{TiO}_{2}$ sont en équilibre au-dessus de $1070 \mathrm{~K}$ ainsi que le gaz environnant. Aux températures intermédiaires, 700-1 000 K, il suppose que :

- les défauts atomiques et électroniques, dans le volume de l'oxyde, sont en équilibre entre eux sans que la surface et le gaz environnant le soient ;

- l'activité de l'oxygène dans l'oxyde est déterminée par la concentration d'oxygène adsorbé.

Un modèle cinétique, traitant de l'adsorption du $\mathrm{CO}$ et de l'oxygène à la surface du matériau, est utilisé pour expliquer le déplacement du point de transition des caractéristiques stationnaires avec la température : $R=f(A / F)$ où $R$ est la résistance de l'oxyde et $A / F$ le rapport Air/Fuel. 
A notre avis, il n'est pas rigoureux de considérer que les défauts dans le volume sont en équilibre entre eux alors que les flux de matière vers une surface hors d'équilibre ne sont pas négligeables; il faut toutefois souligner que l'approximation proposée par Logothetis lui permet d'interpréter ses résultats expérimentaux.

Dans le cas où tout à la fois le volume, la surface et le gaz environnant sont hors d'équilibre, Windischmann [9] et Morisson [10] interprètent la variation de la résistance de $\mathrm{SnO}_{x}$ en $p_{\mathrm{Co}}^{-1 / 2}$ à température peu élevée $(570 \mathrm{~K}) ; 1$ 'oxygène chimisorbé à la surface de l'échantillon est enlevé sous l'action du CO suivant un mécanisme de Rideal-Eley.

Notre contribution est relative aux capteurs résistifs de $\mathrm{TiO}_{2-x}$, en frittés ou en couches, en présence d'atmosphères hors d'équilibre thermodynamique, en régime stationnaire; son originalité consiste à définir la non-stœchiométrie de l'oxyde par des considérations cinétiques.

L'application de ces capteurs étant essentiellement la régulation de la combustion automobile, nous cherchons à obtenir un faible temps de réponse à un changement de $p_{\mathrm{O}_{2}}$. Nous avons prouvé [11], comme d'autres auteurs d'ailleurs [12, 13], que les couches épaisses (sérigraphie, $e \# 25 \mu \mathrm{m}$ ) ou minces (pulvérisation cathodique, $e \# 1 \mu \mathrm{m}$ ) de ce matériau réalisent des capteurs avec des constantes de temps allant respectivement de quelques centaines à quelques dizaines de millisecondes. Nous avons pu obtenir, pour les couches épaisses, en équilibre thermodynamique avec l'atmosphère, des caractéristiques de résistance, $R=f\left(\log p_{\mathrm{O}_{2}}\right)$, tout à fait comparables à celles des structures massives [8], compte tenu du facteur géométrique de nos échantillons et de leur résistivité légèrement plus haute (additif de verre $3 \%$ ).

La catalyse hétérogène est un autre domaine dans lequel sont abordés les interactions gazoxydes dans des processus essentiellement hors d'équilibre thermodynamique. Par exemple, l'oxydation du CO par passage au travers d'une poudre d'oxyde de cérium de grande surface spécifique, à une température relativement peu élevée $(590 \mathrm{~K})$, devra être irréversible pour produire du $\mathrm{CO}_{2}$ [14]. Les auteurs interprètent leurs résultats par le mécanisme suivant :

$$
\begin{aligned}
& k_{3} \\
& \mathrm{CO}(\mathrm{g}) \underset{k_{-3}}{\rightleftarrows} \mathrm{CO}_{\mathrm{ads}} \\
& \mathrm{CO}_{\mathrm{ads}}+\mathrm{O}_{\mathrm{O}}^{x} \rightarrow \mathrm{CO}_{2 \mathrm{ads}}+\mathrm{V}_{\mathrm{O}}^{x} \\
& \mathrm{CO}_{2 \text { ads }} \rightleftarrows \mathrm{CO}_{2}(\mathrm{~g}) \\
& \mathrm{O}_{2}(\mathrm{~g})+2 \mathrm{~V}_{\mathrm{O}}^{x} \rightarrow 2 \mathrm{O}_{\mathrm{O}}^{x} \text {. }
\end{aligned}
$$

« ads » en indice indique des espèces considérées sont adsorbées à la surface de l'oxyde. Les résultats relient la vitesse d'oxydation du $\mathrm{CO}$ à la conduction électronique de l'oxyde en supposant que la concentration des électrons de conduction $\left[\mathrm{e}^{\prime}\right]$ est égale à la concentration $\left[\mathrm{V}_{\mathrm{O}}^{x}\right]$ des lacunes d'oxygène neutres. Herrmann [15] étudie l'oxydation du $\mathrm{CO}$ sur $\mathrm{TiO}_{2-x}$ à des températures de 1'ordre de $720 \mathrm{~K}$. Le mécanisme réactionnel proposé met en œuvre des ions oxygène adsorbés $O_{\text {ads }}^{-}$sans qu'interviennent les défauts atomiques de l'oxyde contrairement au cas de $\mathrm{CeO}_{2-x}$.

$$
\begin{gathered}
\mathrm{O}_{2}(\mathrm{~g})+2 \mathrm{e}^{\prime} \rightarrow 2 \mathrm{O}_{\mathrm{ads}}^{-} \\
\mathrm{CO}_{\mathrm{ads}}+\mathrm{O}_{\mathrm{ads}}^{-} \rightarrow \mathrm{CO}_{2 \text { ads }}+\mathrm{e}^{\prime}
\end{gathered}
$$

La conduction électrique est reliée à la vitesse de la réaction d'oxydation du CO par la concentration des électrons de conduction $\left[\mathrm{e}^{\prime}\right]$. 
Dans l'interprétation de nos expériences, nous nous sommes efforcés de tenir compte des résultats acquis pour les différents types de capteurs, ainsi qu'en catalyse hétérogène, dans l'oxydation de l'oxyde de carbone. Les hypothèses essentielles sur lesquelles nous nous appuierons sont :

- en milieu oxydant (Fig. 1a)

* adsorption d'espèces neutres ;
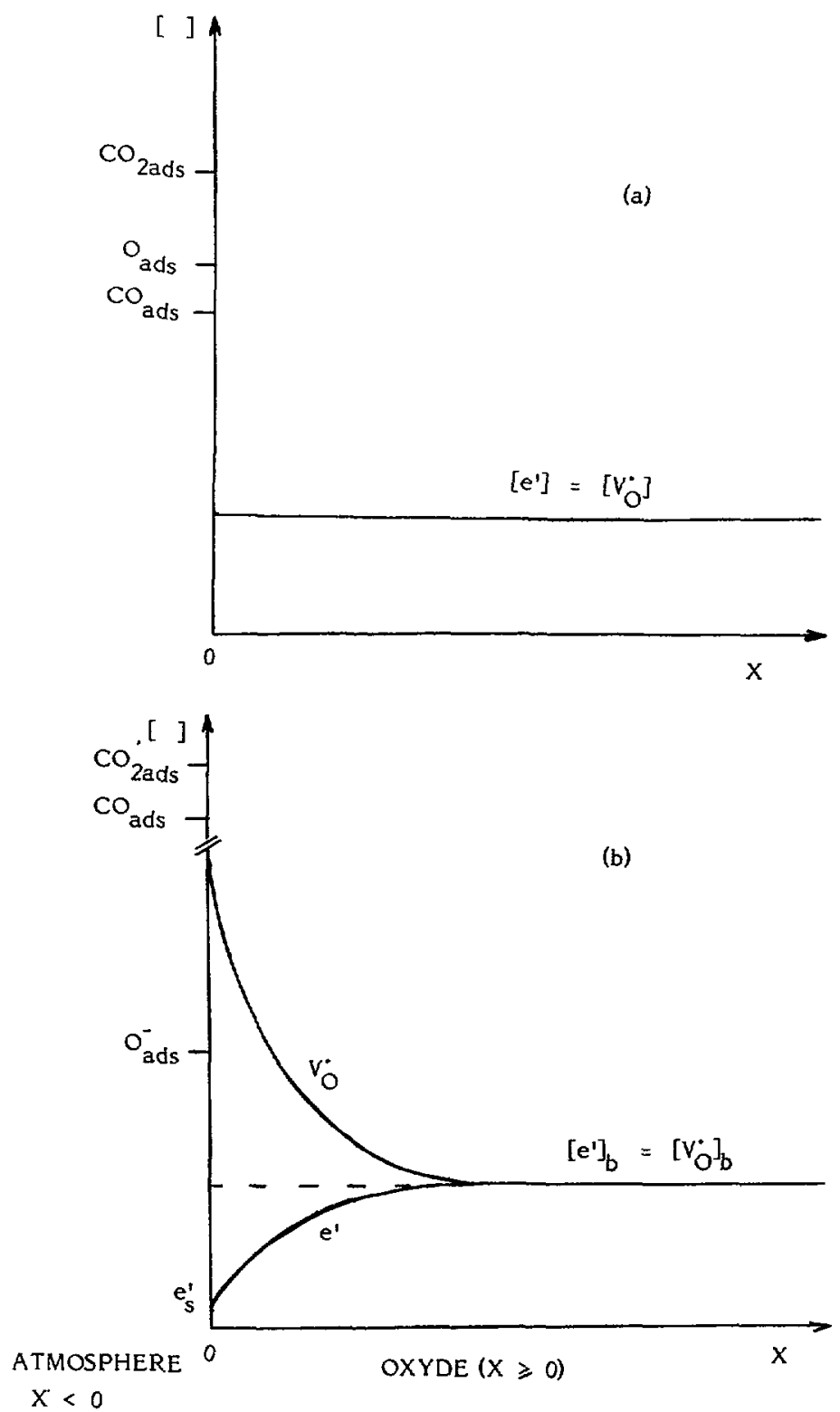

Fig. 1. - Concentration des défauts chargés en fonction de la distance $X$ à la surface : a) milieu oxydant ; b) milieu réducteur.

[Ionised defects concentration versus distance $X$ from the surface : a) oxidizing medium ; b) reducing medium.] 
* double couche négligeable ;

* l'ensemble du matériau est homogène ;

- en milieu réducteur (Fig. 1b)

* adsorption redox conduisant à l'existence d'une couche superficielle chargée négativement ;

* double couche de déplétion réduisant la concentration en électrons au voisinage de la surface.

\section{Matériaux et dispositif expérimental.}

La poudre de $\mathrm{TiO}_{2}$, nécessaire à la fabrication des capteurs, est obtenue par coprécipitation en réalisant l'hydrolyse de l'isopropylate de titane. Le précipité, formé d'hydroxyde de titane, après étuvage, est broyé pour donner une poudre dont les agglomérats ont environ $0,5 \mu \mathrm{m}$ de diamètre. Les échantillons massifs $\mathrm{TiO}_{2}-\mathrm{M}$ (porosité $10 \%$ ) sont préparés par compactage isostatique de la poudre à $400 \mathrm{MPa}$ et frittage à $1370 \mathrm{~K}$. L'addition de bicarbonate d'ammonium lors du compactage permet d'obtenir également des échantillons poreux $\mathrm{TiO}_{2}-\mathrm{PO}$ (porosité $30 \%$, frittage à $1370 \mathrm{~K}$ ).

Les échantillons $\mathrm{TiO}_{2}-\mathrm{M}$ et $\mathrm{TiO}_{2}-\mathrm{PO}$ sont ensuite usinés pour avoir une forme cylindrique (longueur de 10 à $20 \mathrm{~mm}$, diamètre $4 \mathrm{~mm}$ ); les contacts électriques sont réalisés à la laque de platine.

Lés capteurs en couches $\mathrm{TiO}_{2}$-SF sont élaborés par sérigraphie (épaisseur de l'ordre de $25 \mu \mathrm{m}$ avec une cuisson à $1120 \mathrm{~K}$ qui assure notamment la transformation totale d'anatase en rutile [16]). Les électrodes sont en $\mathrm{Pt}$, déposé par pulvérisation cathodique R.F. Leur facteur géométrique est environ $10^{5} \mathrm{~m}^{-1}$ en géométrie coplanaire.

L'observation au microscope électronique à balayage nous apprend que :

- les grains de l'échantillon fritté poreux sont beaucoup plus petits $(\phi=1 \mu \mathrm{m})$ que ceux $(\phi \simeq 5 \mu \mathrm{m})$ de l'échantillon fritté $\mathrm{TiO}_{2}-\mathrm{M}$. Ils sont de plus mal soudés les uns aux autres faisant apparaître des joints de grains très marqués contrairement à $\mathrm{TiO}_{2}-\mathrm{M}$;

- les couches $\mathrm{TiO}_{2}$-SF ont une morphologie, analogue à celle d'une poudre, constituée d'agglomérats $(\phi \# 0,8 \mu \mathrm{m})$ contenant des grains plus petits $(\phi \# 0,2 \mu \mathrm{m})$. Les agglomérats sont nettement différenciés les uns des autres et la surface spécifique est beaucoup plus élevée que celle des frittés.

Les mesures de conductivité électrique sont faites par la méthode des quatre points tant que la résistance est inférieure à $100 \mathrm{k} \Omega$ et au-delà par la méthode des deux points.

L'étude est menée en plaçant les échantillons dans un four étanche dans lequel circule le gaz excitateur à débit variable; le montage expérimental introduit dans le four est représenté sur la figure 2. La référence de la sonde zircone est l'air. Les volumes des constituants du mélange gazeux $\left(\mathrm{O}_{2}, \mathrm{CO}\right.$, Ar) sont dosés par une centrale de contrôleurs de débit massique. Les tensions de consigne ainsi que les acquisitions de mesures sont réalisées automatiquement par calculateur via le Bus IEEE.

Les gaz sont analysés à la sortie du four à l'aide d'un chromatographe à phase gazeuse modèle 5890A de Hewlett Packard:

- le $\mathrm{CO}_{2}$ avec la colonne « chromosorb $10280 / 100 »$;

- le $\mathrm{CO}$ et $\mathrm{O}_{2}$ avec la colonne « molecular sieve $13 \mathrm{X}$ ».

Le calcul des pressions partielles des gaz est effectué à partir des aires des chromatogrammes en tenant compte de la sensibilité des colonnes vis-à-vis des gaz.

La variation du débit des deux gaz réactifs $\mathrm{O}_{2}$ et $\mathrm{CO}$, réalise des régimes stationnaires plus ou moins éloignés de l'équilibre thermodynamique ; l'écart à l'équilibre croît avec le débit. 


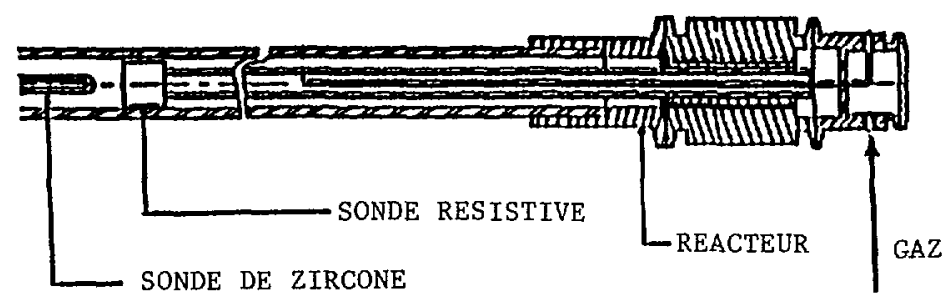

Fig. 2. - Montage expérimental.

[Experimental setup.]

Les débits utilisés vont de 2 à $14 \times 10^{-6} \mathrm{~m}^{3} / \mathrm{s}$ ce qui, compte tenu de la section du four, $9 \times 10^{-4} \mathrm{~m}^{2}$ correspond à des vitesses linéaires allant de 2,2 à $15,6 \times 10^{-3} \mathrm{~m} / \mathrm{s}$. Au-delà des $10^{-5} \mathrm{~m}^{3} / \mathrm{s}$, nous contrôlons le débit avec moins de précision, ce domaine étant en dehors des limites d'utilisation normale des régulateurs.

L'argon ne joue qu'un rôle tampon diminuant le volume des gaz susceptibles de participer à la réaction de combustion. Son pourcentage dans le mélange reste constant et égal à $80 \%$.

Nous appellerons $R_{\mathrm{fg}}$ le rapport $\mathrm{Air} / \mathrm{Fuel}$, simulé par :

$$
R_{\mathrm{fg}}=\frac{p_{\mathrm{CO}}}{p_{\mathrm{O}_{2}}}
$$

\section{Résultats.}

Nous présentons des résultats correspondant à deux températures :

- celle de $870 \mathrm{~K}$ où la combustion du CO n'est pas tout à fait achevée,

- et celle de $1100 \mathrm{~K}$ où elle est quasi-complète et de surcroît rapide.

L'étude de la réponse des capteurs avec la variation du débit du gaz a été menée essentiellement dans deux milieux chimiquement différents :

- un milieu réducteur correspondant à $R_{\mathrm{fg}}=2,4$, à l'équilibre thermodynamique $p_{\mathrm{O}_{2}}=10^{-18} \mathrm{~Pa}$ à $870 \mathrm{~K}$ et $10^{-11} \mathrm{~Pa}$ à $1100 \mathrm{~K}$,

- et un milieu oxydant à $R_{\mathrm{fg}}=1,6\left(p_{\mathrm{O}_{2}} \sim 10^{3} \mathrm{~Pa}\right.$ pour toute température $)$.

La correspondance entre $R_{\mathrm{fg}}$ et $p_{\mathrm{O}_{2}}$ a été établie en considérant que pour $R_{\mathrm{fg}}<2$ tout le monoxyde de carbone est consommé. De même pour $R_{\mathrm{fg}}>2$ c'est pratiquement tout l'oxygène qui est utilisé conformément à la réaction :

$$
\mathrm{CO}+\frac{1}{2} \mathrm{O}_{2} \rightleftarrows \mathrm{CO}_{2}
$$

Des analyses chromatographiques complètent les caractéristiques stationnaires. Cela consiste à identifier et à doser les gaz résiduels à la sortie du four à débit constant. En atmosphère réductrice, lorsque la combustion du $\mathrm{O}_{2}$ est quasi-complète à $1100 \mathrm{~K}$, par exemple, nous ne retrouverons à la sortie que du $\mathrm{CO}$ et du $\mathrm{CO}_{2}$. De même à $R_{\text {fg }}=1,6$, il n'y a que $\mathrm{O}_{2}$ et $\mathrm{CO}_{2}$. A plus basse température, comme $870 \mathrm{~K}$, les analyses montrent la présence des deux gaz actifs pour chaque milieu.

La sonde de zircone est utilisée comme référence aussi sa tension est-elle indiquée sur les figures en même temps que la résistance des différentes structures en $\mathrm{TiO}_{2}$.

2.1 A $870 \mathrm{~K}$. - Figure 3 nous avons rassemblé les réponses (variation de résistance ou de tension) pour trois capteurs en milieu oxydant $\left(R_{\mathrm{fg}}=1,6\right)$ : une sonde de zircone, un 


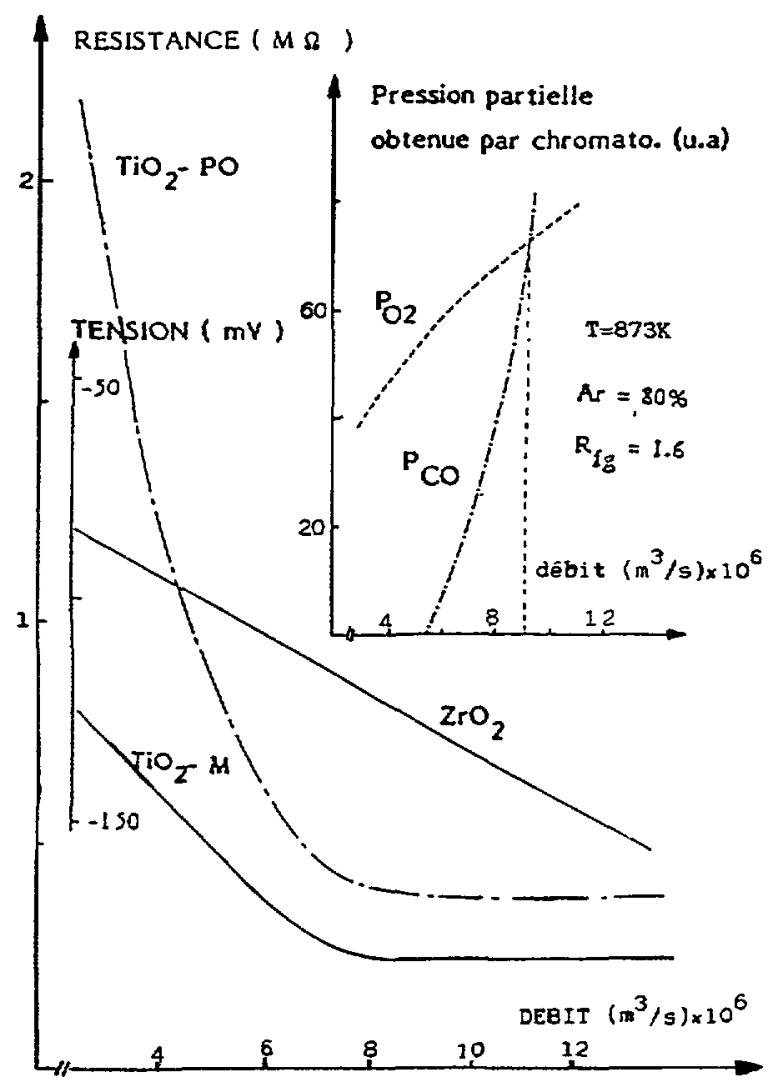

Fig. 3. - Variation de la réponse des capteurs résistifs massifs et de la sonde $\mathrm{ZrO}_{2}$ en fonction du débit à $870 \mathrm{~K}$ et en milieu oxydant; l'analyse des gaz résiduels est indiquée en encadré.

$\left[\mathrm{TiO}_{2}\right.$ sintered ceramics resistance and $\mathrm{ZrO}_{2}$ voltage as a function of the gas flow rate at $870 \mathrm{~K}$ in an oxidizing medium; chromatographic analysis of the gas mixture.]

échantillon massif fritté $\mathrm{TiO}_{2}-\mathrm{M}$ et un échantillon massif fritté poreux $\mathrm{TiO}_{2}-\mathrm{PO}$. La structure poreuse est intermédiaire par sa morphologie entre le massif et la couche épaisse et elle sera utilisée à des fins de comparaison.

La variation de résistance qui accompagne l'accroissement du débit est monotone décroissante atteignant un palier à $7,5 \times 10^{-6} \mathrm{~m}^{3} / \mathrm{s}$. La tension de jauge décroît linéairement ; en première analyse c'est la manifestation d'une faible réduction. La sensibilité des sondes varie avec la composition de l'atmosphère gazeuse.

L'analyse chromatographique de cette dernière est donnée en encadré. Nous avons porté en ordonnée les pressions partielles en unité arbitraire c'est-à-dire l'aire des chromatogrammes corrigée de la sensibilité des colonnes d'analyse. Nous observons l'accroissement rapide de la quantité du $\mathrm{CO}$ dans le mélange, dont la courbe vient couper celle de $p_{\mathrm{O}_{2}}$ à l'abscisse $8,8 \times 10^{-6} \mathrm{~m}^{3} / \mathrm{s}$. Pour ce débit nous retrouvons donc à la sortie du four un rapport $R_{\mathrm{fg}}=1$ alors qu'à l'entrée du four celui-ci était égal à 1,6. C'est la manifestation de la mauvaise combustion de $\mathrm{CO}$ avec l'augmentation du débit.

La figure 4 fournit la réponse de $\mathrm{TiO}_{2}$ en couche $\left(\mathrm{TiO}_{2}-\mathrm{SF}\right)$ en milieu oxydant. La sensibilité de l'échantillon est comparable à celle de la sonde zircone qui indique que la quantité de $\mathrm{CO}$ mise en jeu est inférieure à la quantité représentée figure 3. L'analyse 


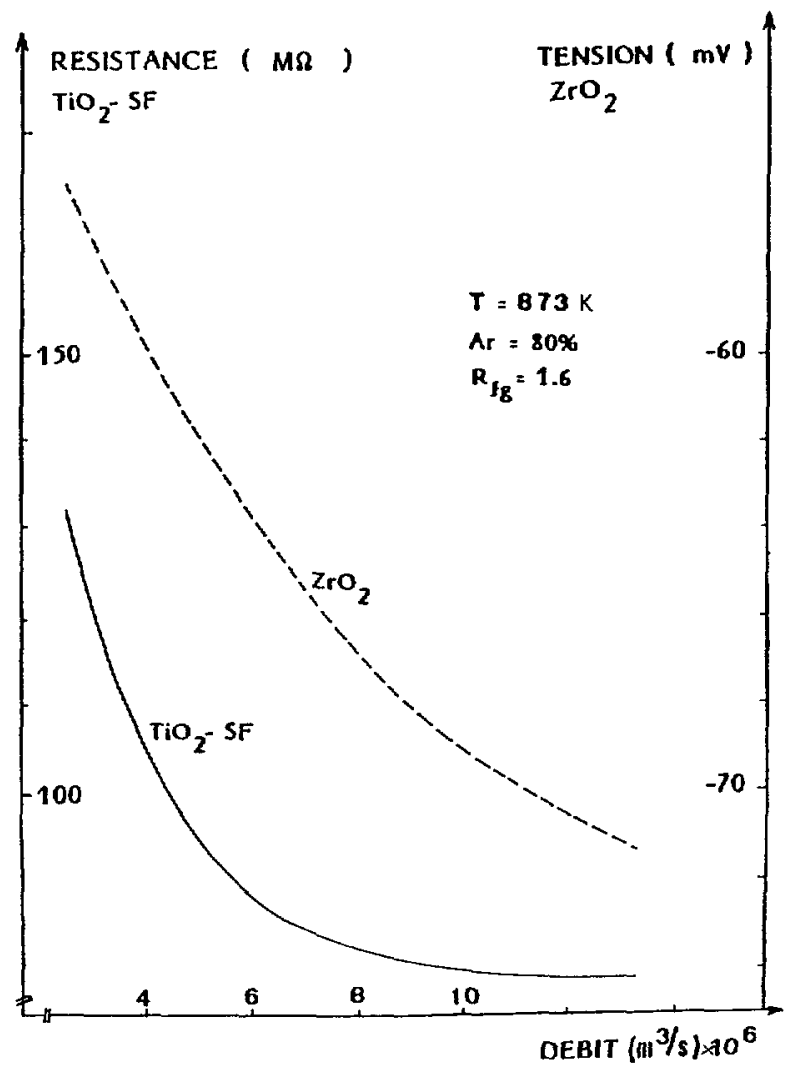

Fig. 4. - Variation de la résistance du capteur $\mathrm{TiO}_{2}-\mathrm{SF}$ et de la tension de la sonde $\mathrm{ZrO}_{2}$ en fonction du débit à $870 \mathrm{~K}$ et en milieu oxydant.

[ $\mathrm{TiO}_{2}-\mathrm{SF}$ film resistance and $\mathrm{ZrO}_{2}$ voltage as a function of the gas flow rate at $870 \mathrm{~K}$ in an oxidizing medium.]

chromatographique ne permet pas dans ce cas de déceler le $\mathrm{CO}$. Nous observons que la résistance de $\mathrm{TiO}_{2}-\mathrm{SF}$ tend vers un palier aux forts débits.

Passons à l'étude en milieu réducteur; il correspond à un rapport $R_{\mathrm{fg}}=2,4$ à l'entrée du four. La variation des résistances et de la tension est ici (Figs. 5 et 6) monotone croissante sauf pour l'échantillon massif dense, $\mathrm{TiO}_{2}-\mathrm{M}$, qui reste insensible à l'accroissement du débit. L'analyse chromatographique montre dans ce cas 1'apparition d'une quantité d'oxygène s'accentuant avec le débit. Par contre les pressions partielles de $\mathrm{CO}$ et $\mathrm{CO}_{2}$ restent constantes; les quantités de $\mathrm{CO}$ et $\mathrm{CO}_{2}$ mises en jeu en milieu réducteur dépassent de plus d'un ordre de grandeur celles relatives à l'oxygène $\left(p_{\mathrm{CO}}>p_{\mathrm{CO}_{2}}>20 p_{\mathrm{O}_{2}}\right)$.

2.2 A $1100 \mathrm{~K}$. - En milieu oxydant toutes les réponses sont indépendantes du débit (Tab. I) et l'analyse chromatographique est en accord avec ce comportement ( $p_{\mathrm{O}_{2}}=$ Cte).

En milieu réducteur la réponse est constante pour tous les capteurs sauf pour $\mathrm{TiO}_{2}-\mathrm{SF}$ en couche dont la résistance croît avec le débit (Figs. 7 et 8). L'analyse chromatographique n'est pas suffisamment sensible pour déceler d'éventuelles traces d'oxygène. 


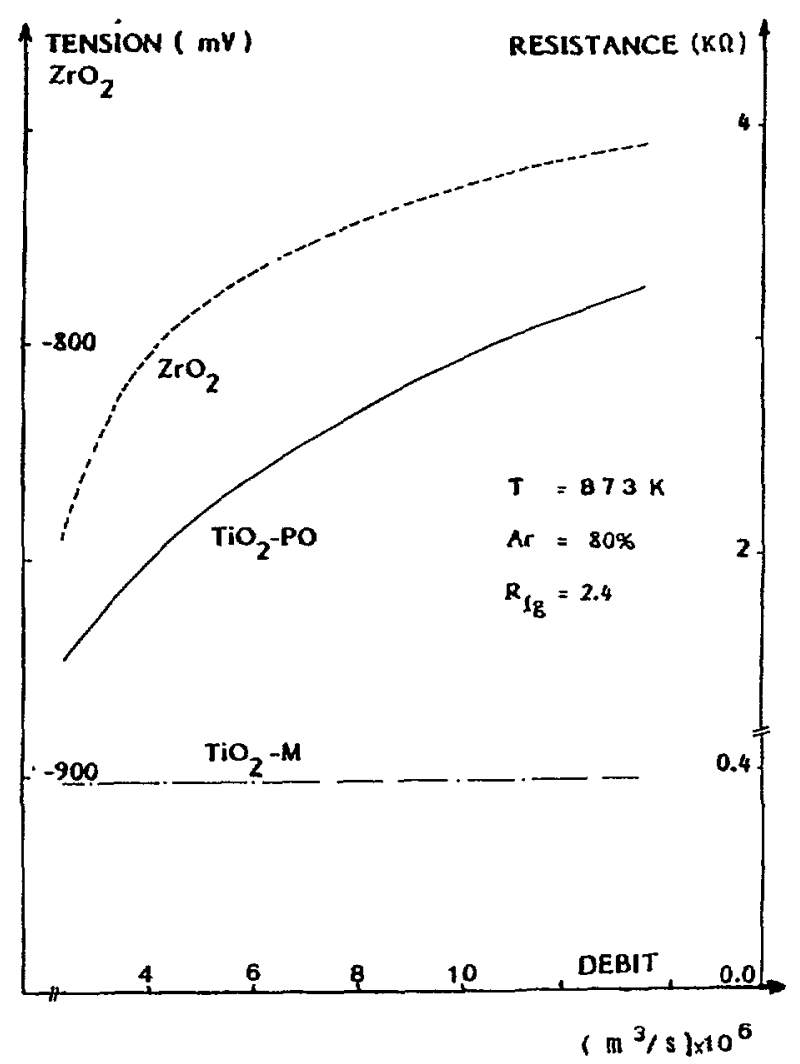

Fig. 5. - Variation de la réponse des capteurs résistifs massifs et de la sonde $\mathrm{ZrO}_{2}$ en fonction du débit, à $870 \mathrm{~K}$ et en milieu réducteur.

$\left[\mathrm{TiO}_{2}\right.$ sintered ceramics resistance and $\mathrm{ZrO}_{2}$ voltage as a function of the gas flow rate at $870 \mathrm{~K}$ in a reducing medium.]

Tableau I. - Réponse des capteurs à $1100 \mathrm{~K}$ en milieu oxydant ( $\left.p_{\mathrm{O}_{2}}=\mathrm{Cte}\right)$. [Sensors responses at $1100 \mathrm{~K}$ in oxiding medium $\left(p_{\mathrm{O}_{2}}=\right.$ Cst.).

\begin{tabular}{|c|c|}
\hline Capteurs & $R_{\mathrm{fg}}$ \\
\hline $\mathrm{ZrO}_{2}$ & 1,6 \\
\hline $\mathrm{TiO}_{2}-\mathrm{SF}$ & $-75 \mathrm{mV}$ \\
\hline $\mathrm{TiO}_{2}-\mathrm{M}$ & $8,7 \mathrm{M} \Omega$ \\
\hline $\mathrm{TiO}_{2}-\mathrm{PO}$ & $35 \mathrm{k} \Omega$ \\
\hline
\end{tabular}




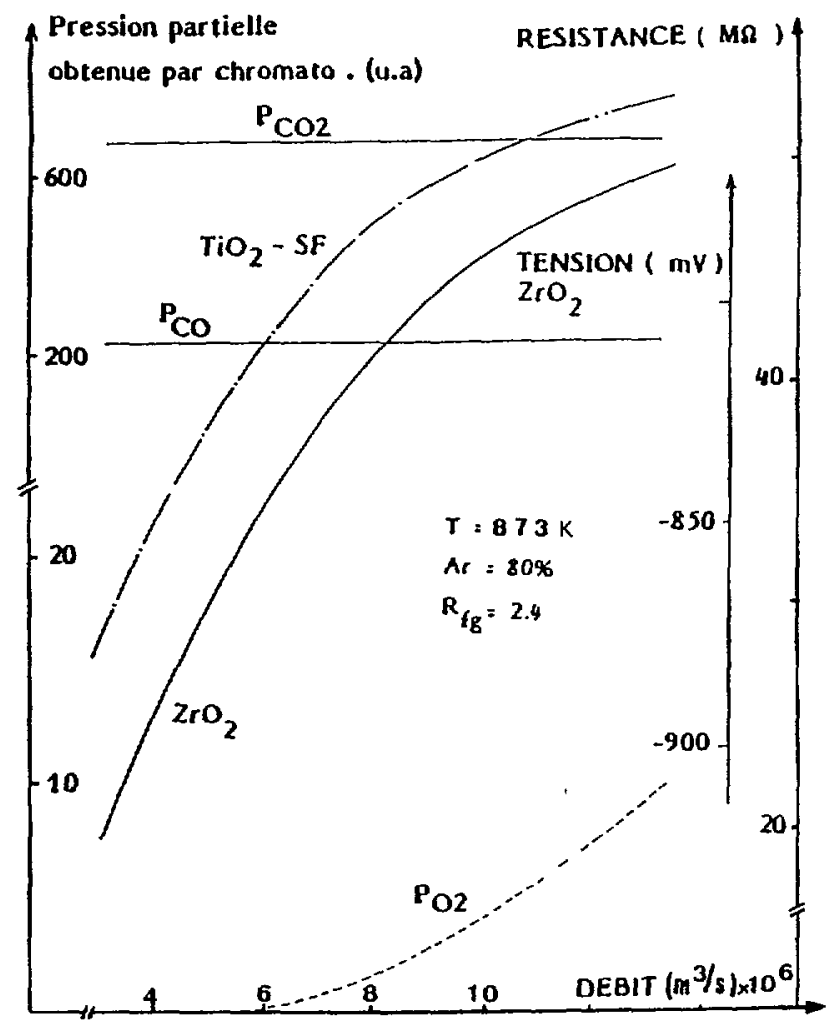

Fig. 6. - Réponses des capteurs $\mathrm{TiO}_{2}-\mathrm{SF}$ et $\mathrm{ZrO}_{2}$ en fonction du débit à $870 \mathrm{~K}$ et en milieu réducteur ; analyse chromatographique des gaz résiduels.

[ $\mathrm{TiO}_{2}-\mathrm{SF}$ and $\mathrm{ZrO}_{2}$ responses as a function of the gas flow rate, at $870 \mathrm{~K}$ and in a reducing medium ; chromatographic analysis of the gas mixture.]

Pour compléter les observations précédentes, il nous faut commenter à nouveau les valeurs des résistances mesurées dans chaque milieu et aux deux températures. Dans ce but, nous donnons les valeurs des résistances à l'équilibre thermodynamique qui serviront de référence (Tab. II et III) :

* A $870 \mathrm{~K}$ côté oxydant les réponses à l'équilibre de tous les capteurs se situent dans le prolongement des courbes obtenues du côté des faibles débits. En particulier les résistances des sondes résistives sont inférieures aux valeurs d'équilibre mais néanmoins assez proches.

* A $870 \mathrm{~K}$ côté réducteur les valeurs d'équilibre se situent aussi du côté des faibles débits. Cependant pour $\mathrm{TiO}_{2}-\mathrm{SF}$, la valeur d'équilibre, égale à $64 \mathrm{k} \Omega$, est nettement éloignée de celle de $20 \mathrm{M} \Omega$ relative au régime stationnaire. L'apparition progressive de l'oxygène en atmosphère réductrice entrâ̂ne une augmentation de la résistance dans le domaine de la dizaine de megohms.

* A $1100 \mathrm{~K}$ en milieu oxydant les capteurs résistifs s'écartent au maximum de $15 \%$ de leur résistance d'équilibre dans le sens d'une réduction. La zircone voit également une atmosphère plus réductrice.

* A $1100 \mathrm{~K}$ en milieu réducteur $\mathrm{TiO}_{2}-\mathrm{PO}$ semble plus oxydé que $\mathrm{TiO}_{2}-\mathrm{M}$, dont la résistance se situe très près de sa valeur d'équilibre. C'est le cas également pour la zircone. 


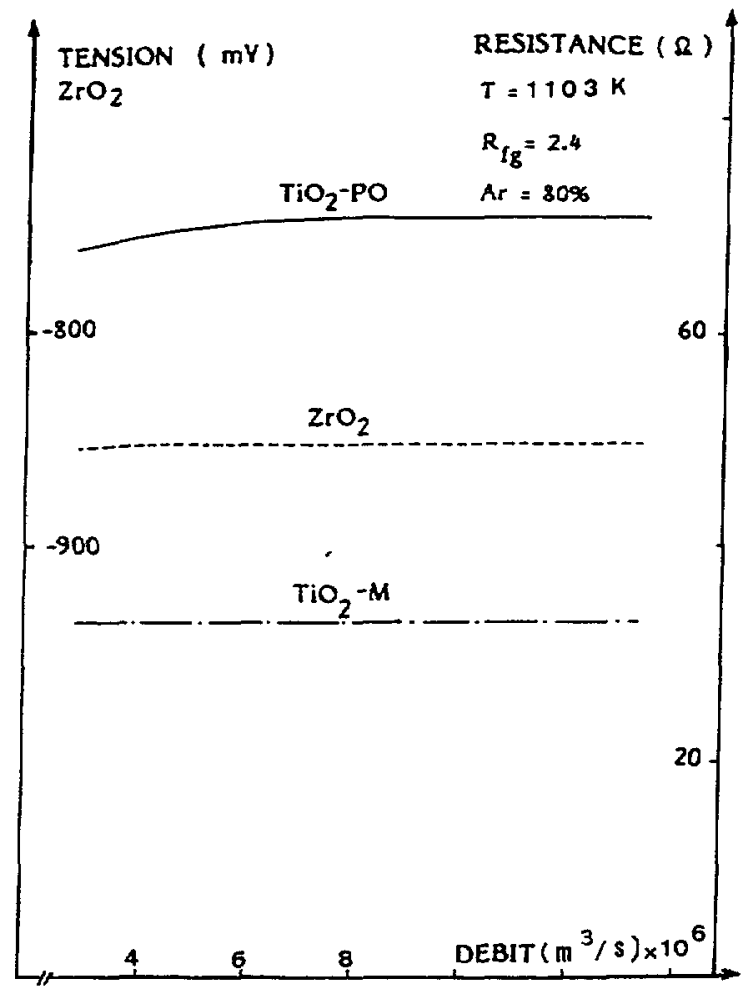

Fig. 7. - Réponses des capteurs résistifs massifs et de la sonde $\mathrm{ZrO}_{2}$ à $1100 \mathrm{~K}$ et en milieu réducteur. $\left[\mathrm{TiO}_{2}\right.$ sintered ceramics and $\mathrm{ZrO}_{2}$ responses as a function of the gas flow rate, at $1100 \mathrm{~K}$, in a reducing medium.]

Tableau II. - Valeurs des résistances des différentes structures en $\mathrm{TiO}_{2}$ à l'équilibre thermodynamique $(870 \mathrm{~K})$.

[Resistance values of the different $\mathrm{TiO}_{2}$ structures when thermodynamic equilibrium is reached $(870 \mathrm{~K})$.]

\begin{tabular}{|c|c|c|}
\hline Capteurs & 1,6 & 2,4 \\
\hline $\mathrm{ZrO}_{2}$ & $-48 m V$ & $-980 \mathrm{mV}$ \\
\hline $\mathrm{TiO}_{2}-\mathrm{SF}$ & $500 \mathrm{M} \Omega$ & $64 \mathrm{k} \Omega$ \\
\hline $\mathrm{TiO}_{2}-\mathrm{M}$ & $2 \mathrm{M} \Omega$ & $300 \Omega$ \\
\hline $\mathrm{TiO}_{2}-\mathrm{PO}$ & $3 \mathrm{M} \Omega$ & $400 \Omega$ \\
\hline
\end{tabular}

L'échantillon $\mathrm{TiO}_{2}$-SF s'approche de sa valeur d'équilibre à faible débit et s'en écarte assez fortement d'un facteur 8 pour les débits supérieurs. Sa sensibilité aux traces d'oxygène est exceptionnelle.

Les caractéristiques Résistance-Pression d'oxygène, mesurées à l'équilibre thermodynami- 


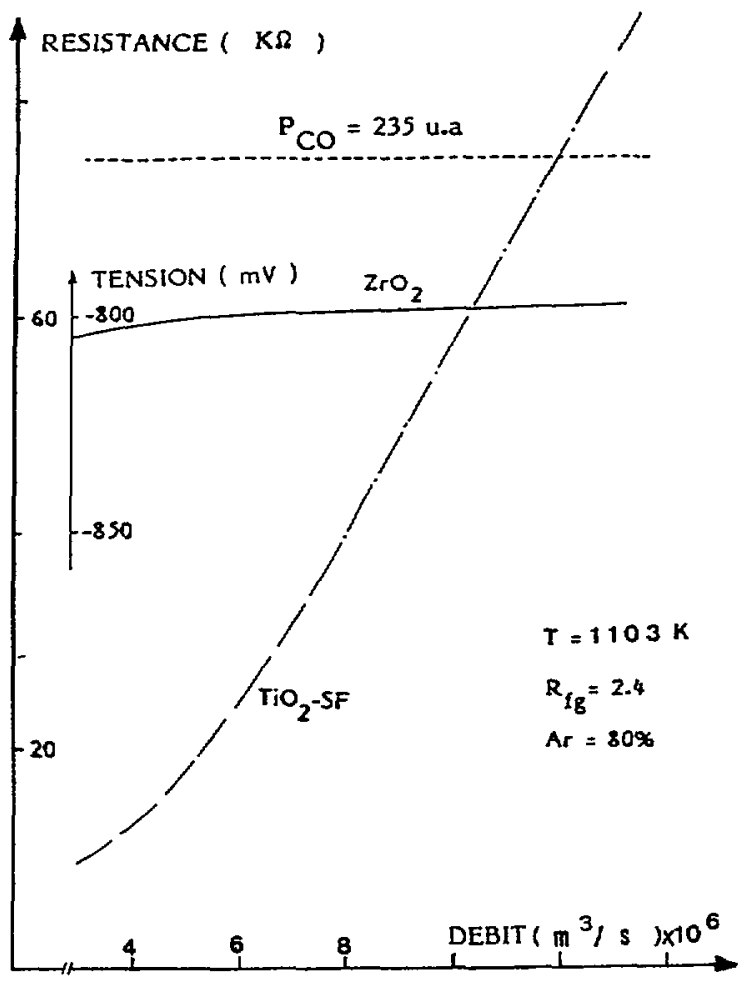

Fig. 8. - Réponses des capteurs $\mathrm{TiO}_{2}-\mathrm{SF}$ et $\mathrm{ZrO}_{2}$ en fonction du débit à $1100 \mathrm{~K}$ et en milieu réducteur; analyse des gaz résiduels $\mathrm{O}_{2}$ et $\mathrm{CO}$.

$\left[\mathrm{TiO}_{2}-\mathrm{SF}\right.$ and $\mathrm{ZrO}_{2}$ responses, $\mathrm{CO}$ partial pressure as a function of the gas flow rate at $1100 \mathrm{~K}$, in a reducing medium.]

Tableau III. - Valeurs des résistances des différentes structures en $\mathrm{TiO}_{2}$ à l'équilibre thermodynamique $(1100 \mathrm{~K})$.

[Resistance values of the different $\mathrm{TiO}_{2}$ structures when thermodynamic equilibrium is reached $(1100 \mathrm{~K})$.]

\begin{tabular}{|c|c|c|}
\hline Capteurs & 1,6 & 2,4 \\
\hline $\mathrm{ZrO}_{2}$ & $-60 \mathrm{mV}$ & $-854 \mathrm{mV}$ \\
\hline $\mathrm{TiO}_{2}-\mathrm{SF}$ & $9 \mathrm{M} \Omega$ & $7 \mathrm{k} \Omega$ \\
\hline $\mathrm{TiO}_{2}-\mathrm{M}$ & $40 \mathrm{k} \Omega$ & $30 \Omega$ \\
\hline $\mathrm{TiO}_{2}-\mathrm{PO}$ & $60 \mathrm{k} \Omega$ & $40 \Omega$ \\
\hline
\end{tabular}

que dans le domaine de température $870-1100 \mathrm{~K}$ par nous-mêmes [11] et Logothétis [8], montrent l'apparition d'une conduction de type $\mathrm{p}$ aux températures les moins élevées $(870 \mathrm{~K})$ pour $p_{\mathrm{O}_{2}} \geqslant 10^{3} \mathrm{~Pa}$. Pour des pressions d'oxygène inférieures à $10 \mathrm{~Pa}$, l'oxyde est de type $\mathbf{n}$ 
(Résistance $\propto p_{\mathrm{O}_{2}}^{1 / 4}$ ) quelle que soit la température $(T \geqslant 870 \mathrm{~K})$. En milieu oxydant, à $870 \mathrm{~K}$, les valeurs de résistance prises au palier, figures 3, 4, par les trois types d'échantillons, correspondraient, à l'équilibre thermodynamique, à une pression d'oxygène de $10^{-6} \mathrm{~Pa}$; ainsi, dès que la présence de CO devient significative, la conduction en milieu oxydant est, soit de type $\mathrm{n}(T=1100 \mathrm{~K})$, soit passe rapidement de type $\mathrm{p}$ au type $\mathrm{n}(T=870 \mathrm{~K})$. En d'autres termes, l'oxyde de titane, dans le domaine de température supérieure à $870 \mathrm{~K}$, en présence d'une atmosphère présentant un gaz oxydant $\left(\mathrm{O}_{2}\right)$ et un gaz réducteur $(\mathrm{CO})$, reste toujours de type $n$; c'est dans cette optique que nous interpréterons nos résultats en régime stationnaire.

\section{Interprétation.}

Nous recherchons, à l'aide des résultats présentés, à établir une expression donnant la résistance des capteurs en fonction des pressions partielles des gaz constituant l'atmosphère, en contact avec leur surface. L'idée que nous poursuivrons consiste à différencier le comportement du capteur en milieu oxydant et en milieu réducteur, et donc à proposer des modèles de conduction distincts tout en adoptant un traitement commun de la concentration des défauts atomiques et électroniques de l'oxyde (type $\mathrm{n}$ ).

Les auteurs Baumard [17], Panis [18], Marucco [19, 20] ont montré que le défaut atomique majoritaire à haute température $(T \geqslant 1370 \mathrm{~K})$ et basse pression d'oxygène $\left(p_{\mathrm{O}_{2}}<10^{-3} \mathrm{~Pa}\right.$ à $1370 \mathrm{~K}$ ) dans le rutile est le titane interstitiel ionisé quatre fois $\left(\mathrm{Ti}_{\mathrm{i}}^{4}{ }^{\circ}\right)$. A des températures moins élevées $(1070 \mathrm{~K} \leqslant T \leqslant 1370 \mathrm{~K})$ et à des pressions d'oxygène plus fortes $\left(p_{\mathrm{O}_{2}} \geqslant 10^{-3} \mathrm{~Pa}\right.$ à $\left.1370 \mathrm{~K}\right)$ le défaut majoritaire serait des lacunes d'oxygène une ( $\left.\mathrm{V}_{\mathrm{O}}^{\prime}\right)$ ou deux fois $\left(\mathrm{V}_{\mathrm{O}}^{2}\right)$ ionisées $[18,19]$. Nous travaillons à des températures encore moins élevées $(870 \mathrm{~K} \leqslant T \leqslant 1100 \mathrm{~K})$ aussi la compensation des lacunes d'oxygène deux fois ionisées par des impuretés acceptrices est-elle à envisager. Toutefois des mesures de conduction électrique et de thermogravimétrie sur des échantillons de différentes puretés, à des températures inférieures à $1170 \mathrm{~K}$, conduisent Marucco et al. [20] à proposer des lacunes d'oxygène une fois ionisée comme défaut majoritaire. Nous adopterons ce point de vue tout en envisageant au cours de la discussion les modifications éventuelles liées à la nature des défauts atomiques.

\subsection{Milieu oxydant.}

3.1.1 Température de $870 \mathrm{~K}$. - Dans cette atmosphère $p_{\mathrm{CO}}$ et $p_{\mathrm{O}_{2}}$ sont de l'ordre de $10^{3} \mathrm{~Pa}$ avec $p_{\mathrm{O}_{2}} \geqslant p_{\mathrm{CO}}$. De plus $p_{\mathrm{O}_{2}}$ est approximativement égale à $\frac{p_{\mathrm{CO}_{2}}}{3}$ avec $p_{\mathrm{CO}_{2}}$ de l'ordre de $10^{+4} \mathrm{~Pa}$. Nous nous trouvons donc dans les conditions de 1'oxydation catalytique hétérogène de l'oxyde de carbone sur les oxydes de type n que nous avons présentée dans l'introduction; les réactions d'échange entre les phases gazeuse et solide sont les réactions (3), (5) et:

$$
\begin{gathered}
\mathrm{O}_{2}(\mathrm{~g}) \underset{k_{-10}}{\stackrel{k_{10}}{\rightleftarrows}} 2 \mathrm{O}_{\mathrm{ads}} \\
\mathrm{O}_{\mathrm{ads}}+V_{\mathrm{O}}+\mathrm{e}^{\prime} k_{13} \mathrm{O}_{\mathrm{O}}^{x} \\
\mathrm{CO}_{\mathrm{ads}}+\mathrm{O}_{\mathrm{O}}^{x}+\stackrel{k_{12}}{\rightarrow} \mathrm{CO}_{2 \mathrm{ads}}+\mathrm{V}_{\mathrm{O}}^{\cdot}+\mathrm{e}^{\prime}
\end{gathered}
$$

L'état stationnaire suppose que les flux de production et de destruction des $V_{O}$ sont égaux, d'où :

$$
k_{12}\left[\mathrm{CO}_{\text {ads }}\right]\left[\mathrm{O}_{\mathrm{O}}^{\mathrm{x}}\right]=k_{11}\left[\mathrm{O}_{\text {ads }}\right]\left[\mathrm{V}_{\mathrm{O}}\right]\left[\mathrm{e}^{\prime}\right]
$$

expression dans laquelle le symbole [i] représente la concentration de l'espèce $i$. 
D'autre part, en état stationnaire, la concentration des espèces est indépendante du temps :

$$
\begin{gathered}
\frac{\mathrm{d}\left[\mathrm{O}_{\mathrm{ads}}\right]}{\mathrm{d} t}=k_{10} p_{\mathrm{O}_{2}}-k_{-10}\left[\mathrm{O}_{\mathrm{ads}}\right]^{2}-k_{11}\left[\mathrm{O}_{\mathrm{ads}}\right]\left[\mathrm{V}_{\mathrm{O}}^{\cdot}\right]\left[\mathrm{e}^{\prime}\right]=0 \\
\frac{\mathrm{d}\left[\mathrm{CO}_{\mathrm{ads}}\right]}{\mathrm{d} t}=k_{3} p_{\mathrm{CO}}-k_{-3}\left[\mathrm{CO}_{\mathrm{ads}}\right]-k_{12}\left[\mathrm{CO}_{\mathrm{ads}}\right]\left[\mathrm{O}_{\mathrm{O}}^{x}\right]=0 .
\end{gathered}
$$

Avec la relation d'électroneutralité : $\quad\left[\mathrm{V}_{\mathrm{o}}^{*}\right]=\left[\mathrm{e}^{\prime}\right]$.

Nous supposons que le flux de désorption de l'oxygène $k_{-10}\left[\mathrm{O}_{\mathrm{ads}}\right]^{2}$ est grand devant le flux de destruction des lacunes d'oxygène en raison de la très faible valeur des concentrations $\left[\mathrm{V}_{\mathrm{O}}\right]=\left[\mathrm{e}^{\prime}\right]$ en régime oxydant et de la valeur assez élevée de $p_{\mathrm{O}_{2}}\left(10^{3} \mathrm{~Pa}\right)$, d'où d'après la relation (14):

$$
k_{10} p_{\mathrm{O}_{2}} \# k_{-10}\left[\mathrm{O}_{\mathrm{ads}}\right]^{2}
$$

Les relations (13), (14'), (15) et (16) permettent le calcul de la concentration des électrons et de la résistance $R_{\mathrm{b}}$ de l'échantillon compte tenu de :

$$
R_{\mathrm{b}} \# \frac{\alpha}{\left[\mathrm{e}^{\prime}\right]} \text { où } \alpha=\mathrm{Cte}>0 .
$$

Il vient alors :

$$
R_{b} \# K\left(\frac{p_{\mathrm{O}_{2}}^{1 / 2}}{p_{\mathrm{CO}}}\right)^{1 / 2} \text { avec } K=\text { Cte }>0 \text {. }
$$

La formule (18) est également obtenue dans le cas de défauts atomiques $V_{O}^{2}$ ou $\mathrm{Ti}_{\mathrm{i}}^{4^{\circ}}$ compensés par des impuretés acceptrices $\mathrm{A}_{\mathrm{T}_{1}}^{2^{\prime}}$ supposées deux fois ionisées $\left(\left[\mathrm{V}_{\mathrm{O}}^{2 *}\right]=\left[\mathrm{A}_{\mathrm{Ti}}^{2^{\prime}}\right]\right.$ ou $\left.2\left[\mathrm{Ti}_{1}^{4}\right]=\left[\mathrm{A}_{\mathrm{Ti}}^{2^{\prime}}\right]\right)$

Nous n'envisageons pas de chimisorption de l'oxygène à la surface des grains de l'oxyde, comme nous le ferons en milieu réducteur, pour deux raisons :

- en milieu oxydant $\left(p_{\mathrm{O}_{2}} \sim 10^{3} \mathrm{~Pa}\right), \mathrm{TiO}_{2}$ est très résistif et, de ce fait, peu apte à chimisorber à sa surface des atomes d'oxygène ionisés $\left(\mathrm{O}_{\text {ads }}^{-}\right)$, ne disposant pas de suffisamment d'électrons ;

- des mesures de conduction faites sous $p_{\mathrm{O}_{2}}=10^{3} \mathrm{~Pa}$ de 670 à $1670 \mathrm{~K}$ ne montrent pas la rupture de pente caractéristique d'une chimisorption (phénomène exothermique) qui apparaîtrait vers les températures peu élevées.

Pour tester la validité de l'expression (18), il faut connaître la loi de variation des pressions partielles avec le débit du mélange gazeux. Dans ce but, nous supposons que le volume du monoxyde de carbone à la sortie du four est soit une fonction linéaire, soit une fonction quadratique de débit. Nous obtenons ainsi, après calcul, figure 9 , les pressions partielles $p_{\mathrm{CO}}$ et $p_{\mathrm{O}_{2}}$. La figure 10 traduit en équivalent résistance cette variation des pressions partielles avec le débit. L'allure obtenue est tout à fait conforme à ce qui a été observé pour les capteurs résistifs dans ce milieu et dépend peu de la loi exacte de variation des pressions partielles avec le débit.

3.1.2. Température $1100 \mathrm{~K}$. - L L'atmosphère est très proche de l'équilibre thermodynamique quel que soit le débit comme le montrent (Tab. I, II et III) les valeurs des résistances et de 


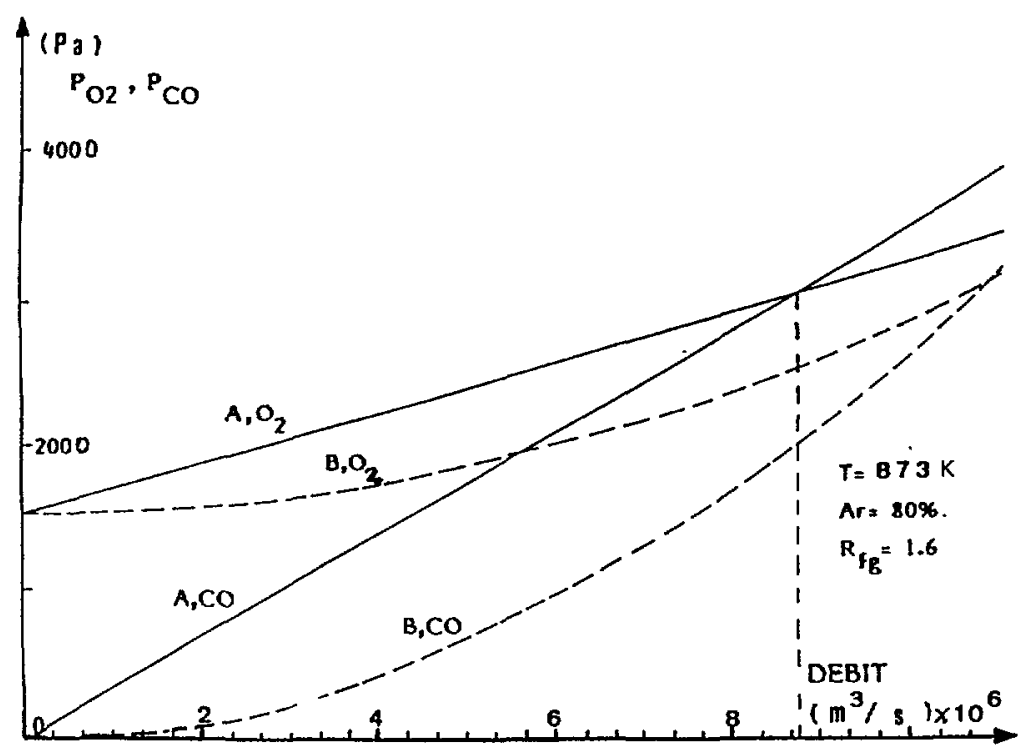

Fig. 9. - Variation des pressions partielles des gaz résiduels $\left(\mathrm{O}_{2}\right.$ et $\left.\mathrm{CO}\right)$ en fonction du débit : A) loi linéaire ; B) loi quadratique.

$\left[\mathrm{O}_{2}\right.$ and $\mathrm{CO}$ calculated partial pressures as a function of the gas flow rate : A) linear law ; B) quadratic law.]

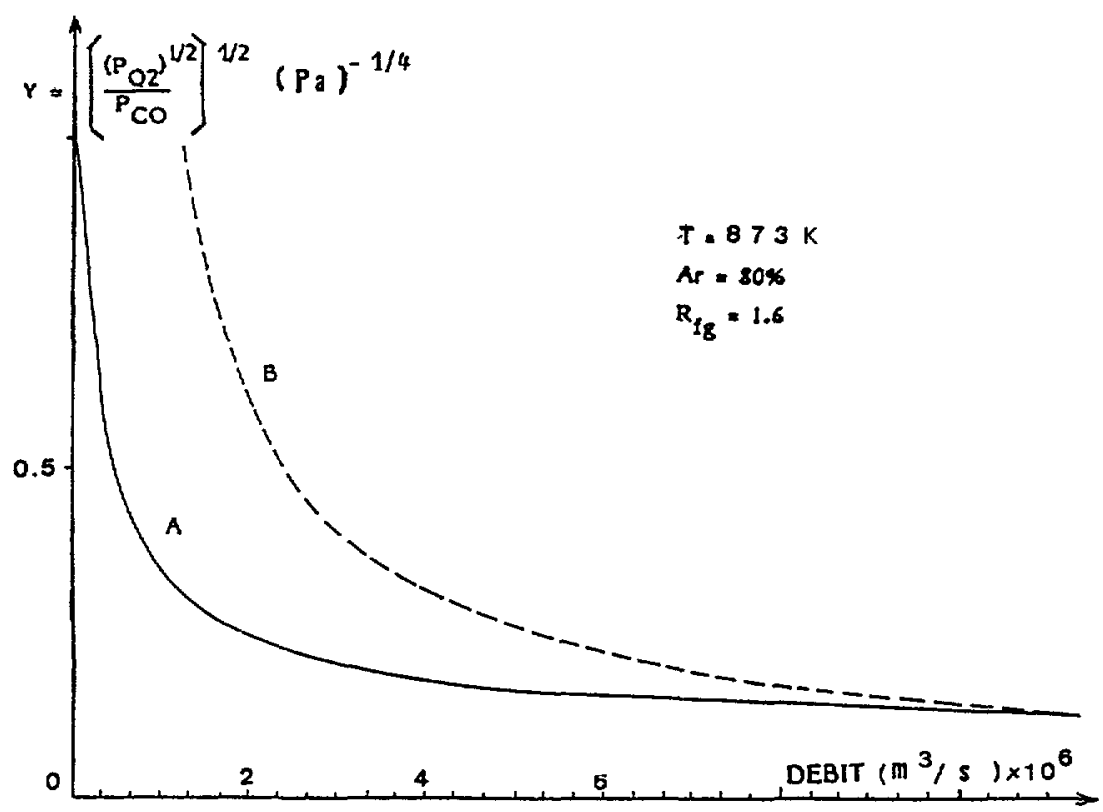

Fig. 10. - Variation du rapport $Y=\frac{\left(p_{\mathrm{O}_{2}}\right)^{1 / 4}}{p_{\mathrm{CO}}^{1 / 2}}$, simulant la variation de résistance, en fonction du débit d'après les pressions partielles calculées de la figure 9 .

[Ratio $Y=\frac{\left(p_{\mathrm{O}_{2}}\right)^{1 / 4}}{p_{\mathrm{CO}}^{1 / 2}}$ as a function of the gas flow rate $Y$ is calculated with the partial pressures plotted in figure 9.] 
tension de la sonde $\mathrm{ZrO}_{2}$. Cet équilibre se traduit par :

$$
\mathrm{O}_{2}(\mathrm{~g})+2 \mathrm{~V}_{\mathrm{O}}+2 \mathrm{e}^{\prime}{ }_{k_{-19}}^{k_{19}} 20_{\mathrm{O}}^{x}
$$

avec :

$$
k_{19}\left[\mathrm{~V}_{\mathrm{O}}^{\cdot}\right]^{2}\left[\mathrm{e}^{\prime}\right]^{2} p_{\mathrm{O}_{2}}=k_{-19}\left[\mathrm{O}_{\mathrm{O}}^{x}\right]^{2}
$$

Il vient :

$$
R_{\mathrm{b}} \# K^{\prime} p_{\mathrm{O}_{2}}^{1 / 4} ; \quad K^{\prime}=\mathrm{Cte}>0
$$

3.2 MiLIEU RÉDUCTEUR. - Avec $R_{\mathrm{fg}}=2,4$, l'atmosphère est presque exclusivement composée de $\mathrm{CO}$ et $\mathrm{CO}_{2}$ dont les pressions partielles sont indépendantes du débit (cf. Fig. 6). En particulier, nous avons $p_{\mathrm{CO}_{2}} \geq 100 p_{\mathrm{O}_{2}}$. Nous pouvons donc considérer que la nonstœchiométrie de l'oxyde est ici entièrement contrôlée par le couple $\mathrm{CO} / \mathrm{CO}_{2} ;$ c'est-à-dire par l'équilibre :

$$
\mathrm{CO}(\mathrm{g})+\mathrm{O}_{\mathrm{o}}^{x}=\mathrm{CO}_{2}(\mathrm{~g})+\mathrm{V}_{\dot{\mathrm{o}}}+\mathrm{e}^{\prime}
$$

par conséquent :

$$
R \# K^{\prime \prime}\left(\frac{p_{\mathrm{CO}_{2}}}{p_{\mathrm{CO}}}\right)^{1 / 2} \text { avec } K^{\prime \prime}=\mathrm{Cte}>0 .
$$

D'après l'expression ci-dessus, la résistance des échantillons ne devrait pas varier avec le débit puisque $p_{\mathrm{CO}}$ et $p_{\mathrm{CO}_{2}}$ sont constantes (Fig. 6); c'est effectivement le cas de $\mathrm{TiO}_{2}-\mathrm{M}$ dès $870 \mathrm{~K}$ (Fig. 5). Nous noterons que la valeur de la rêsistance de $\mathrm{TiO}_{2}-\mathrm{M}$ est très proche de sa valeur à l'équilibre thermodynamique (Tab. II et III). Par contre, à $870 \mathrm{~K}$, la résistance des autres capteurs varie avec le débit (Figs. 5 et 6). A plus haute température $(1100 \mathrm{~K})$ le modèle précédent conviendrait pour expliquer la réponse des capteurs massifs $\mathrm{TiO}_{2}-\mathrm{M}$ et $\mathrm{TiO}_{2}-\mathrm{PO}$ (Fig. 7). Toutefois il est mis en échec par l'échantillon en couche $\mathrm{TiO}_{2}$-SF (Fig. 8) dont la spécificité est d'avoir un rapport surface sur volume plus important que les autres capteurs. Ceci nous conduit tout naturellement à penser que l'action de la surface doit être prise en compte. En effet, en milieu réducteur, les grains de $\mathrm{TiO}_{2-x}$ deviennent très conducteurs ; la concentration élevée d'électrons favorise alors la chimisorption de l'oxygène à leur surface. Ces charges négatives créent des barrières d'énergie potentielle entre grains qui diminuent et contrôlent la conductivité électrique globale de l'échantillon.

En raison de la température assez élevée, nous supposerons que les réactions (7) et (8) sont réversibles. Nous avons observé que la conductivité de $\mathrm{TiO}_{2}-\mathrm{M}$, qui est fixée par $\left[\mathrm{CO}_{\text {ads }}\right]$ et $\left[\mathrm{CO}_{2}\right.$ ads $]$, est indépendante de $p_{\mathrm{O}_{2}}$. La réaction (8), qui modifie ces concentrations avec $\left[\mathrm{O}_{\mathrm{ads}}^{-}\right]$et $p_{\mathrm{O}_{2}}$ doit donc avoir une influence négligeable sur la cinétique de la réaction (7); aussi nous considérerons la réaction de chimisorption de l'oxygène comme un équilibre :

$$
\mathrm{O}_{2}(\mathrm{~g})+2 \mathrm{~S}+2 \mathrm{e}_{\mathrm{s}}^{\prime}=2 \mathrm{O}_{a d s}^{-}
$$

avec

$S \quad$ site superficiel libre

$\mathbf{e}_{\mathrm{s}}^{\prime} \quad$ électron de surface 
ce qui donne :

$$
\frac{\left[\mathrm{O}_{\mathrm{ads}}^{-}\right]}{N_{\mathrm{s}}} \propto p_{\mathrm{O}_{2}}^{1 / 2}\left(1-\frac{\left[\mathrm{O}_{\mathrm{ads}}^{-}\right]}{N_{\mathrm{s}}}\right) \frac{\left[\mathrm{e}_{\mathrm{s}}^{\prime}\right]}{N_{\mathrm{c}}}
$$

avec

$N_{c}$. densité effective d'états par $\mathrm{m}^{3}$

$N_{\mathrm{s}}$. nombre de sites superficiels par $\mathrm{m}^{2} \sim 10^{19} \mathrm{~m}^{-2}$

D'où la résistance $R_{\mathrm{s}}$ de la couche due essentiellement aux joints de grains et aux effets de surface :

$$
R_{\mathrm{s}} \# \frac{\alpha}{\left[\mathrm{e}_{\mathrm{s}}^{\prime}\right]}=K^{\prime \prime \prime} \frac{1-\theta}{\theta} \cdot p_{\mathrm{O}_{2}}^{1 / 2}
$$

avec

$$
\theta(\text { taux de recouvrement })=\frac{\left[\mathrm{O}_{\mathrm{ads}}^{-}\right]}{N_{\mathrm{s}}}
$$

$$
K^{\prime \prime}=\text { Cte }>0
$$

Cette relation suggère que dans le cas d'un taux de recouvrement variant peu avec le débit, la résistance doit varier en $p_{\mathrm{O}_{2}}^{1 / 2}$ C'est ce qui a été observé à $870 \mathrm{~K}$, figure 11 , où la pente de la droite dans un diagramme log-log est très proche de $\frac{1}{2}$.

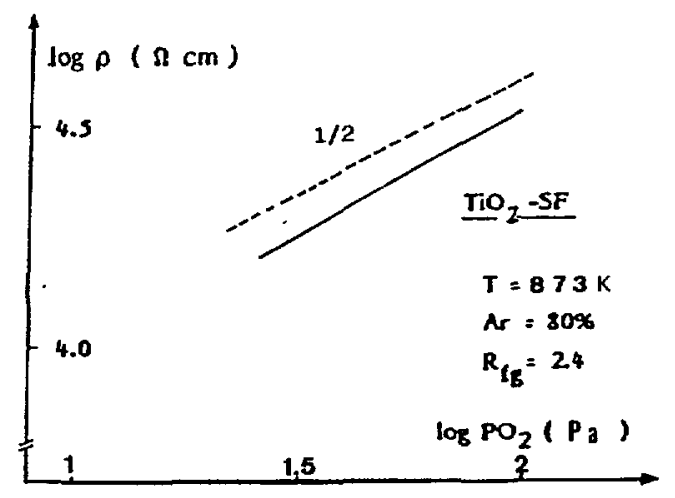

Fig. 11. - Variation de la résistivité des films $\mathrm{TiO}_{2}-\mathrm{SF}$ à $870 \mathrm{~K}$ en fonction de la pression partielle d'oxygène d'après les résultats de la figure 6.

[Dependence of the $\mathrm{TiO}_{2}-\mathrm{SF}$ film resistivity on oxygen partial pressure; $p_{\mathrm{O}_{2}}$ is obtained by chromatographic analysis (Fig. 6).]

Cette vérification expérimentale du modèle peut être confortée en calculant le taux de recouvrement en fonction du débit.

Nous avons effectué ce calcul en supposant que la charge superficielle au niveau de chaque grain était négative et suffisamment faible. Dans ce cas les électrons sont repoussés par cette charge et il s'établit une région de désertion. La courbure des bandes est obtenue en résolvant l'équation de Poisson écrite comme suit :

$$
-\frac{\mathrm{d}^{2} V}{\mathrm{~d} x^{2}}=\frac{q}{\varepsilon}\left(\left[\mathrm{V}_{\mathrm{o}}\right]-\left[\mathrm{e}^{\prime}\right]\right)
$$


avec $\left[\mathrm{e}^{\prime}\right]$ et $\left[\mathrm{V}_{\dot{O}}\right]$ les concentrations à la distance $x$ de la surface du matériau

$$
\varepsilon_{\mathrm{r}}\left(\mathrm{TiO}_{2}\right)=100 ; \quad \varepsilon=\varepsilon_{\mathrm{r}} \varepsilon_{0}
$$

Ces concentrations peuvent s'exprimer à l'aide du potentiel $V$, à l'abscisse $x$, celui du volume étant pris égal à zéro :

$$
\begin{gathered}
{\left[\mathrm{e}^{\prime}\right]=\left[\mathrm{e}^{\prime}\right]_{\mathrm{b}} \exp \frac{q V}{k T} ;} \\
{\left[\mathrm{V}_{\mathrm{o}}\right]=\left[\mathrm{V}_{\dot{\mathrm{o}}}\right]_{\mathrm{b}} \exp \frac{-q V}{k T} ; V<0, q>0 .}
\end{gathered}
$$

L'électroneutralité du volume implique $\left[\mathrm{e}^{\prime}\right]_{\mathrm{b}}=\left[\mathrm{V}_{\dot{\mathrm{o}}}\right]_{\mathrm{b}}$. Nous avons considéré que les lacunes d'oxygène étaient ionisées une fois. Cette hypothèse ne limite pas les conclusions tout en facilitant la résolution de l'équation (23). Nous en déduisons l'expression du taux de recouvrement :

$$
\theta=-\frac{\varepsilon}{q N_{s}} \sqrt{\frac{8 k T[\mathrm{e}]_{b}}{\varepsilon}} \operatorname{sh} \frac{q V_{s}}{2 k T}
$$

où $V_{s}$ est le potentiel de surface.

Pour calculer $\theta$, nous avons besoin de connaître le potentiel de surface dont l'ordre de grandeur peut être obtenu à partir de la relation (24)

avec

$$
V_{\mathrm{s}} \# \frac{k T}{q} \log \frac{R_{\mathrm{b}}}{R_{\mathrm{s}}}
$$

$$
R_{\mathrm{e}} \# R_{\mathrm{b}} \text {. }
$$

En régime stationnaire, $R_{\mathrm{b}}$ est différent de $R_{\mathrm{e}}$ où $R_{\mathrm{e}}$ est la résistance de l'échantillon $\mathrm{TiO}_{2}$-SF en équilibre thermodynamique. Néanmoins, en milieu réducteur, la réaction (21) est pratiquement la seule à contrôler la non-stœchiométrie de l'oxyde et, dans ce cas, nous avons $R_{\mathrm{b}}=R_{\mathrm{e}}$ (cf. Figs. 5 et 6 et Tab. II et III). La concentration d'électrons en volume $\left[\mathrm{e}^{\prime}\right]_{\mathrm{b}}$ est calculée à partir de la valeur de $R_{\mathrm{e}}$, en prenant une mobilité électronique de $10^{-5} \mathrm{~m}^{2} /$ V.s. (21).

Nous obtenons (Tab. IV) des valeurs de $V_{s}$ d'un ordre de grandeur raisonnable qui décroît en valeur absolue lorsque la température s'élève. Il en est de même du taux de recouvrement $\theta$ comme on pouvait s'y attendre puisque la chimisorption de l'oxygène est un phénomène exothermique. Notre hypothèse d'un taux de recouvrement $\theta$ variant peu à $870 \mathrm{~K}$ avec le débit se trouve confirmée ce qui correspond au résultat expérimental d'une résistance variant en $p_{\mathrm{O}_{2}}^{0,5}$. Toutefois, la légère variation de $\theta$ conduit d'après la formule (18'") à une variation de la résistance calculée en $p_{\mathrm{O}_{z}}^{0,3}$. L'écart constaté avec la loi expérimentale peut être attribué au fait que la formule (27) qui donne le potentiel de surface n'est qu'approchée. Par ailleurs, le modèle classique des semi-conducteurs où les donneurs (ici $V_{o}$ ) sont immobiles, et donc en nombre constant, dans I'approximation de la désertion totale dans la zone de charge d'espace, conduit à $\theta$ variant de $0,98 \%$ à $1 \%$ lorsque le débit varie de 5 à $10 \times 10^{-6} \mathrm{~m}^{3} / \mathrm{s}$. La formule $\left(18^{\prime \prime \prime}\right)$ donne alors une variation de la résistance calculée en $p_{\mathrm{O}_{2}}^{0,5}$. Ce dernier modèle correspond parfaitement à la variation de résistance observée expérimentalement ; il est donc de ce point de vue plus satisfaisant que le modèle précédent mais il est critiquable sur le plan théorique car les lacunes d'oxygène ne peuvent être considérées comme immobiles à $870 \mathrm{~K}$ comme le montre la variation de la résistance en $p_{\mathrm{O}_{2}}^{1 / 4}$ dans le cas de l'équilibre thermodynami- 
Tableau IV. - Potentiel de surface et taux de recouvrement en fonction de la température et du débit.

[Surface potential and coverage rate as a function of temperature and gas flow rate.]

\begin{tabular}{|c|c|c|c|c|c|}
\hline \multicolumn{2}{|c|}{$\begin{array}{c}\text { Débit } \\
\left(\mathrm{m}^{3} / \mathrm{s}\right) \times 10^{6}\end{array}$} & 5 & 6,7 & 8,3 & 10 \\
\hline \multirow{3}{*}{$T=870 \mathrm{~K}$} & $\begin{array}{c}p_{\mathrm{O}_{2}} \\
(\mathrm{~Pa})\end{array}$ & 11 & 24 & 41 & 70 \\
\cline { 2 - 6 } & $V_{\mathrm{s}}(\mathrm{V})$ & $-0,48$ & $-0,49$ & $-0,5$ & $-0,5$ \\
\cline { 2 - 6 } & $\theta \%$ & 9,5 & 10 & 10,5 & 11 \\
\hline \multirow{3}{*}{$T=1100 \mathrm{~K}$} & $V_{\mathrm{s}}(\mathrm{V})$ & $-0,09$ & $-0,12$ & $-0,17$ & $-0,20$ \\
\cline { 2 - 6 } & $\theta(\%)$ & 1,3 & 1,7 & 2,6 & 3,2 \\
\hline
\end{tabular}

que. Il faut toutefois souligner qu'à $870 \mathrm{~K}$, les équilibres sont longs à s'établir pour les échantillons massifs, de l'ordre de $1 \mathrm{~h}$, ce qui indique une migration peu rapide des lacunes d'oxygène. Nous en concluons que la situation réelle doit être intermédiaire aux deux modèles développés.

\section{Conclusion.}

Les écarts à la stœechiométrie présentés par l'oxyde de titane en présence d'atmosphère hors d'équilibre thermodynamique et composées de $\mathrm{CO}, \mathrm{O}_{2}, \mathrm{CO}_{2}$ et $\mathrm{Ar}$ ont été étudiés dans le domaine de température $(870-1100 \mathrm{~K})$; ces atmosphères sont d'autant plus éloignées de l'équilibre que le débit est élevé.

Nous avons montré que les variations de résistance des échantillons, en fonction du débit, dépendent du domaine de pression d'oxygène, de la température et de la morphologie des échantillons.

L'utilisation de l'analyse chromatographique des gaz et l'étude des caractéristiques citées conduisent aux conclusions suivantes.

A $870 \mathrm{~K}$ : dans le domaine oxydant :

- la non-stœchiométrie en volume de l'oxyde est déterminée par la cinétique des échanges avec la phase gazeuse et correspond à une variation de la résistance du matériau en $p_{\mathrm{O}_{2}}^{1 / 4} / p_{\mathrm{CO}}^{1 / 2}$

dans le domaine réducteur:

- la non-stœchiométrie en volume de $\mathrm{TiO}_{2-x}$ est fixée par le couple redox $\mathrm{CO} / \mathrm{CO}_{2}$ comme si l'atmosphère était en quasi-équilibre thermodynamique ;

- la chimisorption de l'oxygène contrôle la résistance des couches d'oxyde selon une loi en $p_{\mathrm{O}_{2}}^{1 / 2}$ en créant des barrières de potentiel à la surface des grains ;

- les variations de résistance des échantillons qui sont croissantes avec le débit dans l'ordre massif - poreux - couche indiquent que la surface a une influence prépondérante. La sensibilité à l'oxygène est accrue par une morphologie qui à l'échelle macroscopique (échantillons poreux ou en couches) favorise la diffusion de l'oxygène et qui à l'échelle 
microscopique permet la création de barrière de potentiels aux joints de grains (grande surface spécifique, joints de grains importants). Les modèles développés permettent le calcul du potentiel de surface lié à la chimisorption de l'oxygène et de retrouver la loi expérimentale à $870 \mathrm{~K}$ de la résistance des couches en fonction de la pression d'oxygène. La comparaison des modèles indique une diffusion relativement lente des lacunes d'oxygène à $870 \mathrm{~K}$.

A $1100 \mathrm{~K}$, l'atmosphère tend à être à l'équilibre thermodynamique :

- en atmosphère oxydante, tous les capteurs sont en équilibre avec la pression partielle d'oxygène ;

- en atmosphère réductrice, dominée par le couple redox $\mathrm{CO} / \mathrm{CO}_{2}$, seule la couche $\mathrm{TiO}_{2}$-SF est sensible à la présence de trace d'oxygène.

D'une manière générale, nous pouvons conclure que la non-stœchiométrie d'oxyde en présence d'une atmosphère hors équilibre thermodynamique est déterminée par la cinétique des échanges avec l'atmosphère gazeuse. Dans certaines situations, tout se passe comme si l'atmosphère était en quasi-équilibre thermodynamique mais alors la morphologie des échantillons peut exalter des phénomènes de surface et modifier leur résistance.

\section{Remerciements.}

Nous remercions la Société SFERNICE (Nice, France) qui a préparé les couches de $\mathrm{TiO}_{2}$ par sérigraphie.

\section{Bibliographie}

[1] Fleming W. J., Physical principles governing non ideal behavior of the zirconia oxygen sensor, Electrochem. Sci. Technol. 124 (1977) 21.

[2] FLeming W. J., Zirconia oxygen sensors : origin of non ideal behavior, Proceedings of the 8th Automotive Mat. Conf., Michigan, Published by Am. Ceram. Soc. (29 nov. 1975) p. 272.

[3] Fleming W. J., Device model of the zirconia oxygen sensor, S.A.E. Trans 8602 (1978) p. 1716.

[4] ANDERSON J. E. et Graves Y. B., Stade-state characteristics of oxygen concentration cell sensors subjected to non equilibrium gas mixtures, Electrochem. Sci. Technol. 128 (1981) 294.

[5] Anderson J. E. et Graves Y. B., Hysteresis effects with $\mathrm{ZrO}_{2}$ oxygen sensors exposed to nonequilibrium oxygen-hydrocarbon mixtures, J. Appl. Electrochem. 13 (1983) 395.

[6] Fouletier J., Nouvelles méthodes d'étude des cellules à oxyde électrolyte solide à l'aide des jauges et pompes à oxygène, Thèse de Doctorat, Grenoble (1976).

[7] SALAuN H., Etude du fonctionnement d'un capteur à oxygène dans des mélanges gazeux $\mathrm{CO}-\mathrm{CO}_{2}-\mathrm{O}_{2}$ hors équilibre thermodynamique pour la régulation d'une combustion, Thèse Docteur Ingénieur, Grenoble (1985).

[8] Logothetis E. M., Resistive-type exhaust gas sensors. Proceedings of the 8 th Automotive Mat. Conf., Michigan, Published by Am. Ceram. Soc. (29 nov. 1975) p. 281.

[9] WindischmanN H., MARK P., A model for the operation of a thin-film $\mathrm{SnO}_{x}$ conductancemodulation carbon monoxide sensor, Solid State Sci. Technol. 126 (1979) 627.

[10] MorRISON S. R., Mecanism of semiconductor gas sensor operation, Sensors Actuators 11 (1987) 283.

[11] JeRISIAN R., Gautron J. et Loup J. P., Capteurs d'oxygène à base d'oxydes métalliques, contrat MIR, n ${ }^{\circ} 84 T 0146$ du 25.01.84.

[12] Cook J. A., Hamburg D. R., Kaiser W. J. et Logothetis E. M., Engine dynanometer Study of the transient response of $\mathrm{ZrO}_{2}$ and $\mathrm{TiO}_{2}$ exhaust gas oxygen sensors, J.A.E. Trans. 9203 (1984) 3259.

[13] Hamburg D. R., Cook J. A., Kaiser W. J. et Logothetis E. M., An engine-dynanometer study of the A/F compatibility between a three-way catalyst and an exhaust gas oxygen sensor, J.A.E. Trans. 9203 (1984) 3273. 
[14] Breysse M., Guenin M., Claudel. B., Latrenle H., Veron J., Catalysis of carbon monoxide oxidation by Cerium dioxide. I correlations between catalytic activity and electrical conductivity, J. Catal. 27 (1972) 275.

[15] Heermann J. M., Latreille H., Vergnon P., Veron J., Teichner S., Mise en évidence d'une forme active de l'oxygène dans la réaction d'oxydation du monoxyde de carbone par la mesure de la conductivité électrique du bioxyde de titane (anatase) en cours de catalyse, J. Chim. Phys. 6 (1974) 943.

[16] Bondar IZV AKAD NAUK SSR I. A., Phase transitions in $\mathrm{TiO}_{2}$, Neorganiche Skie Materialy 7 (1971) 1183.

[17] Baumard J. F., Défauts et propriétés électriques du rutile pur ou dopé à haute température (1 200-1 $800 \mathrm{~K}$ ), Thèse de doctorat, Orléans (1977).

[18] PANIS D., Relation entre la conductivité électrique et les défauts dans le rutile réduit entre 1250 et $1750 \mathrm{~K}$, Thèse $3^{\circ}$ cycle, Orléans (1976).

[19] Marucco J. F., Gautron J., Lemasson P., Loup J. P., Corrélation entre les propriétés de $\mathrm{TiO}_{2-x}$ en équilibre à $1100^{\circ} \mathrm{C}$ et après trempe, C.R. Acad. Sci. Paris 289 (1979) C117.

[20] Marucco J. F., Gautron J., Lemasson P., Thermogravimetric and electrical study of nonstoichiometric titanium dioxyde $\mathrm{TiO}_{2-x}$ between 800 and $1100^{\circ} \mathrm{C}, J$. Phys. Chem. Solids 42 (1981) 363.

[21] Williams D. E. et MC Geehin P., Solid-state gas sensors and monitors, Specialist Period. Rep. Electrochem. 9 (Royal Society of Chemistry, 1984) 246. 